\title{
Calculating the Curie Temperature reliably in diluted III-V ferromagnetic semiconductors
}

\author{
Georges Bouzerar ${ }^{1 *}$, Timothy Ziman ${ }^{1 \dagger}$ and Josef Kudrnovský ${ }^{2 \ddagger}$ \\ ${ }^{1}$ Institut Laue Langevin BP 15638042 Grenoble France. \\ ${ }^{2}$ Institute of Physics, Academy of Sciences of the Czech Republic,Na Slovance 2,CZ-182 21 \\ Prague 8, Czech Republic
}

(November 20, 2018)

\begin{abstract}
We present a semi-analytic theory for the Curie temperature in diluted magnetic semi-conductors that treats disorder effects exactly in the effective Heisenberg Hamiltonian, and spin fluctuations within a local RPA. The exchange couplings are taken from concentration dependentab initio estimates. The theory gives very good agreement with published data for wellannealed samples of $\mathrm{Mn}_{x} \mathrm{Ga}_{1-x} \mathrm{As}$. We predict the critical temperatures for $\mathrm{Mn}_{x} \mathrm{Ga}_{1-x} \mathrm{~N}$ lower than in doped GaAs, despite the stronger nearestneighbour ferromagnetic coupling. We also predict the dependence on the hole concentration.
\end{abstract}

*email:bouzerar@ill.fr

†and CNRS, email:ziman@ill.fr

‡email:kudrnov@fzu.cz 
Search for diluted magnetic semiconductors with ferromagnetism stable to high temperatures has been hampered by the many physical parameters that may determine magnetic properties. These include the choice of host semiconductor, that of the doping magnetic impurity, the degree of compensation, and methods of preparation and treatment of the sample [1]. The underlying mechanism of interaction between dopant spins has without doubt been correctly identified: Ruderman-Kittel-Kasuya-Yosida(RKKY)-like effective interactions mediated by both the host $[2,3]$ and the doping band [4-6]. Despite this, the theory has not lead to reliable quantitative predictions. Comparison of calculations has been complicated by difficulties of full characterizing the samples. In samples of GaAs doped with Mn, extensive experimental studies [1] have now allowed for greater control over sample parameters and there is now apparently convergence to reliable experimental values of the critical temperature of different groups [7-10]. An important factor was that the carrier densities were measured simultaneously by magneto-transport. There is now the possibility of testing calculations against experiments and determining the origin of past discrepancies.

Ab initio calculations using the Local Density Approximation and the magnetic force theorem can be used [5] to derive realistic values of magnetic exchange interactions between classical impurity spins. These calculations also take into account the effect on the effective exchange of disorder of the carriers, within a Coherent Potential Approximation (CPA). Similar calculations based on supercells $[12,13]$ lead to comparable results at low concentration. It has become apparent that the difficulty is not in deriving the effective magnetic Hamiltonian correctly but in treating its thermodynamics accurately. As we will demonstrate explicitly here, treating the magnetic correlations by over-simplified mean field theories $[14,15]$ has lead to overstatement, by a wide margin, of the critical temperature $\mathrm{T}_{c}$. The disorder in the effective magnetic model also plays an important role that cannot be simply treated by an effective medium theory of the style of the Virtual Crystal Approximation (VCA) [16]. This suggests that the discrepancy with experiment is largely due to approximations made to the effective Hamiltonian, not the values of the couplings themselves. Thus by improving the treatment of the effective Hamiltonian we can hope to 
find good agreement with experiments.

In this paper we shall argue that the effective random Heisenberg model may be treated by an accurate semi-analytic method separating the treatment of the disorder, which will be treated without approximation, and an analytic approach, a form of the Random Phase Approximation (RPA) for spin fluctuations. Thus the calculation starting from first principles is in three stages: first the $a b$ initio calculation of the effective Heisenberg couplings with pairs of magnetic dopants at different relative displacements. In the second stage, we generate a sequence of different configurations on the fcc lattice by sampling techniques. For each configuration the random Heisenberg model is treated analytically within RPA. This approximation is an extension of standard RPA of the Heisenberg model to a disordered system. It is equivalent to that used in ref [16], except in that case the disorder was treated in a CPA-type manner. As the lattice configuration is random, the equations must be solved numerically. The full derivation of the equations will be given elsewhere [17]: here we shall simply summarize the determination of the the critical temperature: The Green's functions $G_{i j}(E)$ for spins on impurity sites $i$ and $j$, satisfy

$$
\begin{aligned}
E G_{i j}(E)= & 2 \lambda_{i} \delta_{i j}+\left(\sum_{l} J_{l j} \lambda_{l}\right) G_{i j}(E) \\
& -\epsilon\left[\lambda_{i} \sum_{l} J_{i l} G_{l j}(E)\right]
\end{aligned}
$$

where the variables $\lambda_{i}$ are the average magnetization on individual sites, normalized with respect to the magnetization averaged over all impurities. For an RPA treatment of Heisenberg spins the value of $\epsilon$ is 1 . In order to compare to approximations that we shall term "Ising-like", this term can be neglected, ie $\epsilon$ taken to be zero. For a given temperature the Green's functions for impurity spins are determined following a self-consistent procedure for the $G_{i j}(E)$ and $\lambda_{i}(T)$ similar to that of Callen $[18,16]$. In the limit of $\mathrm{T} \rightarrow \mathrm{T}_{c}$ and a total of $N_{0}$ classical spins, we can write:

$$
F_{i}=-\frac{1}{2 \pi \lambda_{i}} \int_{-\infty}^{\infty} \frac{\operatorname{Im} G_{i i}(E)}{E} d E
$$




$$
k_{\mathrm{B}} \mathrm{T}_{\mathrm{c}}=\frac{2}{3 N_{0}} \sum_{i} \frac{1}{F_{i}}
$$

$\mathrm{T}_{c}$ is now determined by the self consistency of these equations, which are solved exactly for a given configuration. The critical temperatures are averaged over different samples (typically $10^{5}$ host sites averaged over 50 configurations). We remark that the results are close to recent results obtained by a Monte Carlo simulations [19], which also take into account fluctuations in the positions of the magnetic impurities. Semi-analytical calculations are intrinsically much faster and essentially no finite-size extrapolation is needed. For a given configuration, equations 2 and 3 give an explicit form of $\mathrm{T}_{c}$. The greatly improved accuracy over previous mean field methods is due essentially to the fact that the RPA approximation includes the low temperature modes that destroy long range order as the temperature increases, and that the geometric disorder is fully included. 


\section{FIGURES}

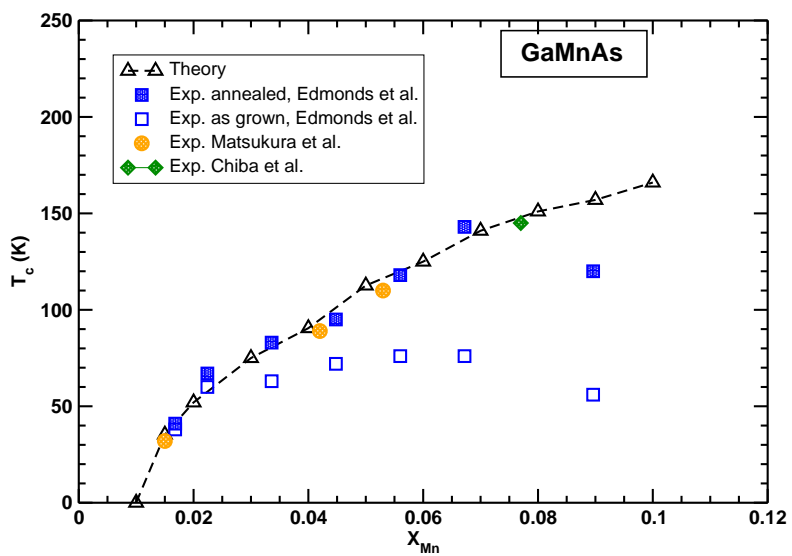

FIG. 1. $\mathrm{T}_{c}$ as a function of doping for $\mathrm{Mn}_{x} \mathrm{Ga}_{1-x}$ As: theory for uncompensated samples and experiment.

In Figure 1 we show the calculation of $\mathrm{T}_{c}$ as a function of $\mathrm{x}$ for $\mathrm{Mn}_{x} \mathrm{Ga}_{1-x} \mathrm{As}$. We show also the experimental results of Edmonds et al [7,8], Matsukura [9], and Chiba et al. [10] for different concentrations of of $\mathrm{Mn}$ in $\mathrm{Mn}_{x} \mathrm{Ga}_{1-x} \mathrm{As}$. We note that the agreement with the results of Edmonds et al. is for the case of fully annealed samples. This is consistent with the fact that we use the couplings calculated for uncompensated samples. The annealing changes the compensation via the density of Mn interstitials and As antisites. This also increases the density of carriers, as shown by transport studies. The agreement with experiment is excellent, except for the single highest concentration (9\%): our theory suggests that at this concentration annealing is not complete. Note also that the theory correctly predicts a threshold ( about 1.5\%) below which there is no ferromagnetism. 


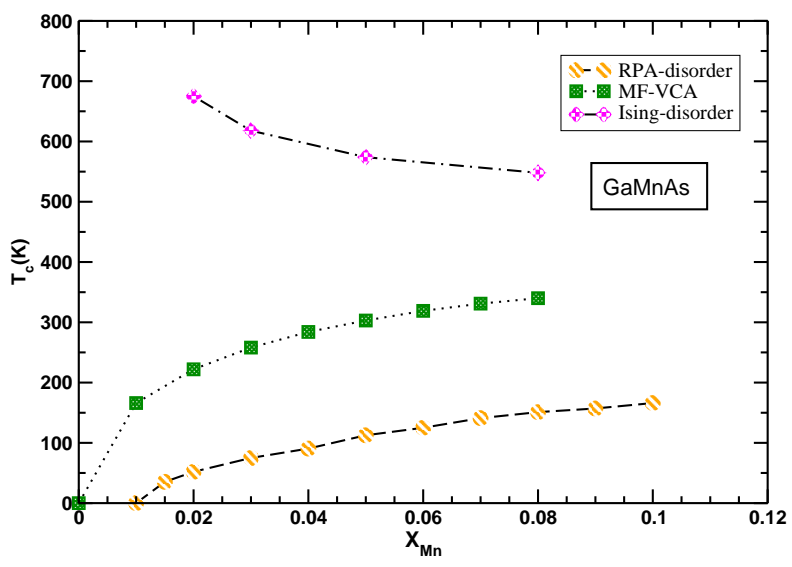

FIG. 2. $\mathrm{T}_{c}$ for $\mathrm{Mn}_{x} \mathrm{Ga}_{1-x} \mathrm{As}$ : comparison of different theories, as defined in the text.

In Figure 2 we show the calculations of $\mathrm{T}_{c}$ as a function of $\mathrm{x}$ for $\mathrm{Mn}_{x} \mathrm{Ga}_{1-x}$ As within different theoretical approaches: "Ising" like Mean Field theory $(\epsilon=0$ in equation 1, but fully including disorder), , Mean Field-Virtual Crystal Approximation (MF-VCA) in which the disorder is treated as a simple effective medium: $T_{c}^{M F-V C A}=\frac{2}{3} x \sum_{i} J_{0 i}$, RPA-disorder $(\epsilon$ $=1$, full disorder and transverse fluctuations, the present approach). One can see the large over-estimate of the critical temperatures for the "Ising-like" mean field approximations (essentially the same as in ref [15] ) which includes the disorder correctly but does not treat transverse fluctuations. In such simple mean field theories the critical temperature is overly influenced by sites in large local effective fields due to strong short-range ferromagnetic interactions. For low dilution long-range order cannot propagate simply by nearest neighbour interactions. The RPA form, in contrast, gives more weight to the low frequency excitations and this is the reason for its success. The MF-VCA results reproduce essentially results of Sato et al. [14], showing that the difference with our final theory is in the treatment of the effective Hamiltonian, not in the couplings estimated $a b$ initio. The current theory and Monte-Carlo simulations [19] show the threshold effect for ferromagnetism: this is an example of the failure of the simplest RKKY-like theories.

The flexibility and accuracy of our calculation allows us to make more precise the ques- 
tion of the dependence of the Curie temperature on the density of carriers. In Figure 3 we present the estimated critical temperature as a function of the number of carriers for the dopant concentration 5\%. For direct experimental comparison, annealing may also change the number of magnetically active impurities through the variation of the density of Mn interstitials. For simplicity we analyse the effect of carrier density assuming a constant magnetic impurity density. The exchange integrals are calculated by introducing compensation via a concentration $y$ of As antisites, giving a carrier density $\mathrm{n}_{h}=x-2 y$. It is seen that the temperature is rather insensitive to hole density provided that it is above a value of about $60 \%$ of the number of dopant atoms. Below this value it diminishes rapidly and ferromagnetism disappears between 50 and $60 \%$. From the dependence of the coupling constants on concentration $x$ ( see Figure 5a of ref. [5]) we expect the threshold value of $\gamma$ to decrease further as $x$ increases. This transition is associated with suppression of the ferromagnetism presumably in favour of a spin-glass phase, owing to dominance of antiferromagnetic superexchange. For comparison, we show the results if the disorder is treated by a Virtual Crystal Approximation (MF-VCA). While that approximation gave a threshold level of carrier density at rather lower values, it increasingly overestimates the critical temperature as $\gamma$ increases, ie the compensation decreases.

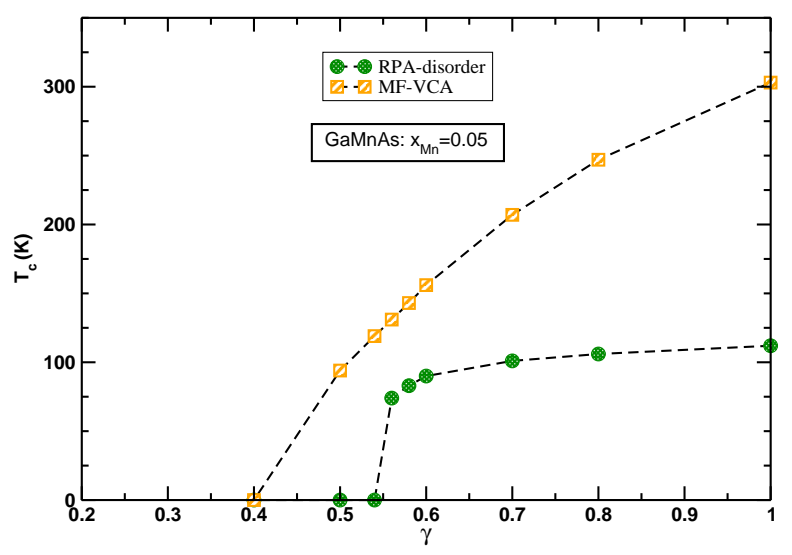


FIG. 3. $\mathrm{T}_{c}$ for $\mathrm{Mn}_{x} \mathrm{Ga}_{1-x} \mathrm{As}$ for fixed concentration as a function of carrier density. The parameter $\gamma$ is the ratio of carrier concentration $\mathrm{n}_{h}$ to the doping density $\mathrm{x}$.

It would be interesting to verify this curve experimentally in different samples. The reference [ [7] ] estimates that in as-grown samples $\gamma$ is about 0.5 for $5 \% \mathrm{Mn}$. Annealing increased $T_{c}$ substantially: this is consistent with our estimates of the transition region. We note that older, possibly less precise, measurements of carrier concentrations [9] gave lower values $(\gamma \approx 0.2)$ where we would predict that ferromagnetism is unstable. This lower value has been used in RKKY-type models which failed to predict the spin-glass instability. This was due to the neglect of superexchange contributions important especially at low carrier concentration [11]. This is in contrast to the ab initio calculations where they are fully included. The relative insensitivity with the degree of compensation indicates that our previous calculations ( Figure 1) should remain accurate in presence of a small number of antisite substitutions. We remark that Figures 1 to 3 indicate the qualitative and quantitative failures of simplified RKKY approaches in which $\mathrm{T}_{c} \propto x^{\frac{4}{3}} \gamma^{\frac{1}{3}}$. While this has already been noted experimentally [8] we can see from a theoretical point of view while this must be so: each exchange in the effective Hamiltonian is renormalized by the disorder, disorder in the effective Hamiltonian is significant, and transverse spin fluctuations are crucial.

We now turn to the calculations made by the same procedure for the case of (GaMn)N which has attracted great interest [20]. Application of simple RKKY models predicted a $\mathrm{T}_{c}$ as high as $700 \mathrm{~K}$ for moderately low concentrations (6\% Mn) [21]. The experimental situation is somewhat controversial: there have been reports of ferromagnetism at high temperatures [22] as well as reports of paramagnetism down to low temperatures [23]. Here we shall content ourselves with estimating the critical temperatures for well-annealed samples. In Figure 4 we show predicted Curie temperatures for $\mathrm{Mn}_{x} \mathrm{Ga}_{1-x} \mathrm{~N}$ showing, for comparison, the same calculations for $\mathrm{Mn}_{x} \mathrm{Ga}_{1-x}$ As. It is seen that the critical temperature of the doped GaN is always lower than for the same concentration of doped GaAs. This is despite the fact 
that the nearest neighbour ferromagnetic coupling is substantially stronger: propagation of long range magnetic order naturally depends crucially on the further nearest neighbour couplings which are much weaker [5]. The reason that they fall off more rapidly is that the impurity band of $\mathrm{Mn}$ is near mid-gap, in contrast to the case of $(\mathrm{Mn}, \mathrm{Ga})$ As where it is close to the valence band edge of GaAs. As in GaAs, the estimates by Sato et al [14] are qualitatively different because of the mean field treatment, with maximum critical temperatures for GaN at $350 \mathrm{~K}$, much higher than the current results. We emphasise that the this difference is not because of the difference in the exchange couplings, but in the subsequent treatment of the effective Heisenberg model.

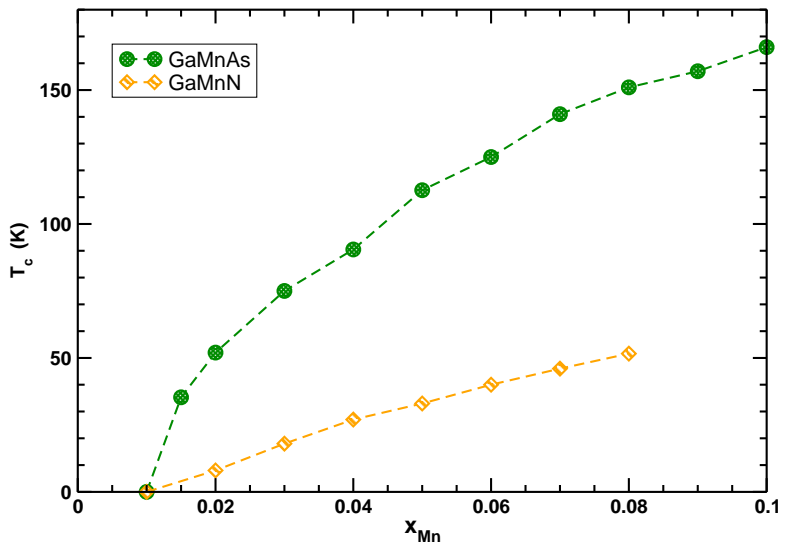

FIG. 4. Predicted $\mathrm{T}_{c}$ for $(\mathrm{Ga}, \mathrm{Mn}) \mathrm{N}$ compared to $(\mathrm{Ga}, \mathrm{Mn}) \mathrm{As}$.

In conclusion, we present a novel approach allowing for reliable calculation, starting from ab initio methods, of the critical temperatures in diluted magnetic semi-conductors. This shows that apparent discrepancies in the past between predictions from ab initio methods and observed material properties has been due to the incomplete treatment of the effective Hamiltonian rather than inaccuracy in the effective exchange couplings, especially because of the need to properly include the effects of disorder. The speed and flexibility of the method allows us to consider parameters that may be useful in designing useful materials in a variety of non-translationally invariant situations. Good agreement is found with experimental 
data on well annealed samples of $\mathrm{Mn}_{x} \mathrm{Ga}_{1-x} \mathrm{As}$. The apparent strong increase of $\mathrm{T}_{c}$ due to proper treatment of disorder, as observed in previous "Ising-like" mean field theories, is shown to be due to neglect of transverse fluctuations. These in fact reduce the critical temperatures significantly compared to simple MF-VCA. We also predict the dependence on the carrier concentration, corresponding to samples with different concentrations of antisites. For the average dopant concentrations chosen, this predicts weak dependence on antisite concentration when this is small but eventually the critical temperature falls rapidly. For doped samples of GaN our theory predicts consistently lower critical temperatures than for GaAs despite the larger short range ferromagnetic coupling. Our estimates differ by an order of magnitude from previous estimates in the concentration range $0-10 \%$. These results call for explicit experimental verification and also show the over-simplification of previous theories.

We would like to thank Dr. K. Edmonds for providing unpublished additional data concerning measured critical temperatures of (GaMn)As. JK acknowledges the financial support from the Grant agency of the Academy of Sciences of the Czech Republic (A1010203 ) and the Czech Science Foundation 202/04/0583). 


\section{REFERENCES}

[1] H. Ohno, Science 281,951 (1998)

[2] T. Dietl, H. Ohno, and F. Matsukura, Phys. Rev. B 63, 195205 (2001)

[3] T. Jungwirth, W. A. Atkinson, B. H. Lee, and A. H. MacDonald, Phys. Rev. B 59, 9818 (1999)

[4] H. Akai, Phys. Rev. Lett. 81, 3002 (1998)

[5] J. Kudrnovský, I. Turek, V. Drchal, F. Maca, P. Weinberger, P. Bruno Phys. Rev. B 69, $115208(2004)$

[6] G. Bouzerar, J.Kudrnovský and P. Bruno, Phys. Rev. B 68, 205311 (2003)

[7] K. W. Edmonds, K.Y. Wang, R.P. Campion, A. C. Neumann, C.T. Foxon, B.L. Gallagher, P.C. Main, Appl. Phys. Lett. 81, 3010 (2002)

[8] K. W. Edmonds et al, Phys. Rev. Lett. 92,037201 (2004), K. W. Edmonds K.Y. Wang, R.P. Campion, B.L. Gallagher, C.T. Foxon, Appl. Phys. Lett. 81, 4991 (2002). Additional values of $\mathrm{T}_{c}$ were provided by Edmonds et al ( private communication).

[9] F. Matsukura, H.Ohno, A. Shen, and Y. Sugawara, Phys. Rev. B 57, R2037 (1998)

[10] D. Chiba, K. Takamura, F. Matsukura, H.Ohno, Appl. Phys. Lett., 82, 3020 (2003)

[11] G. Bouzerar, Phys. Rev. Lett. 92, 069701 (2004)

[12] M. van Schilfgaarde and O.N. Mryasov, Phys. Rev. B 63, 233205 (2001)

[13] L.M. Sandratskii and P. Bruno, Phys. Rev. B 67, 214402 (2003)

[14] K. Sato, P. H. Dederichs, and H. Katayama-Yoshida, Europhys. Lett. 61, 403 (2003)

[15] M. Berciu and R. N. Bhatt, Phys. Rev. B 69 , 045202 (2004), M. Berciu and R. N. Bhatt, Phys. Rev. Lett. 87, 107203 (2001) 
[16] G. Bouzerar and P. Bruno, Phys. Rev. B 66 , 014410 (2002)

[17] G. Bouzerar et al.(unpublished)

[18] H.B. Callen, Phys. Rev. 130, 890 (1963)

[19] P.H. Dederichs, private communications. L. Bergqvist, O. Eriksson, J, Kudrnovský, V. Drchal,P. Korzhavyi, I. Turek, (unpublished).

[20] T. Dietl, H. Ohno, F. Matsukura,J. Cibert,D. Ferrand, Science 2871019 (2000)

[21] T. Dietl, F. Matsukura and H.Ohno, Phys. Rev. B 66, 033203 (2002)

[22] S. Sonoda, H. Hori, Y. Yamamoto, T. Sasaki, M. Sato, S. Shimizu, K. Suga, and K. Kindo, IEEE T. Magn. 38, 2859 (2002),cond-mat/0205560

[23] S. Kuroda, E. Bellet-Amalric, R. Giraud, S. Marcet, J. Cibert, and H.Mariette, Appl. Phys. Lett. 83, 4580 (2003); R. Giraud et al, Europhys. Lett. 65 553(2004) 